\title{
Producción de Sentidos sobre Asistencia Transespecífica en Salud, Derechos y Ciudadanía Trans*
}

\author{
Claudia Leticia Lazcano ${ }^{1}$ \\ ${ }^{1}$ Faculdade Cesusc, SC, Brasil.
}

\author{
Maria Juracy Filgueiras Toneli ${ }^{2}$ \\ ${ }^{2}$ Universidade Federal de Santa Catarina, SC, Brasil.
}

\begin{abstract}
Resumen: En este artículo discutiremos el proceso de producción de sentidos sobre la asistencia transespecífica en salud y su relación con los derechos y la ciudadanía trans*, lo que desarrollamos a partir del diálogo con 11 personas, entre usuarias/os, médicas/os y funcionarias/os de un servicio de atención a personas trans* en una capital en la región Sur de Brasil. Se realizaron entrevistas con las/os participantes, y el análisis de la información se efectuó a partir de la problematización recursiva. Los resultados se organizan en torno a dos tramas de sentidos. Por un lado, se analizan la significación y la potencia de las demandas realizadas por las personas trans* al campo de la asistencia transespecífica en salud, apuntando su relación con una ética comprometida con lo que es excluido por los regímenes de verdad y con la garantía de nuevos flujos de inteligibilidad. Por otro, se analizan las exigencias para constituir un espacio de atención en salud para personas trans* como un ámbito de concretización de la ciudadanía trans*, que supone garantizarse no solo por el acceso al sector de la salud, sino también por las características de la oferta, su formalización, manutención e institucionalización del cuidado a las personas trans*.
\end{abstract}

Palabras clave: Transexualidad, Diversidad de género, Accesibilidad a los servicios de salud, Derechos humanos, Políticas públicas antidiscriminación.

\section{Production of Meanings in Trans-specific Assistance for Health, Rights, and Trans* Citizenship}

\begin{abstract}
In this article we discuss the process of production of meanings around trans-specific health care and its relationship with trans* rights and citizenship, which we develop from a dialogue with 11 people, between users, doctors, and employees of a health service for trans* people located in a capital city in southern Brazil. We interviewed the participants and the analyzed the information based on recursive problematization. The results are organized around two webs of meanings. On the one hand, we analyze the significance and power of the demands made by trans* people in the field of trans-specific health care, pointing out their relationship with an ethic committed to what is excluded by the regimes of truth and with the guarantee of new streams of intelligibility. On the other hand, we analyze the requirements for the constitution of the health care space for trans* people as an area of concretization of trans* citizenship, which is meant to be assured not only by access to the health sector, but also by the characteristics of the offer, its formalization, the maintenance and the institutionalization of care for trans* people.
\end{abstract}

Keywords: Transsexuality, Gender diversity, Accessibility to health services, Human rights, Public anti-discrimination policies. 


\title{
Produção de Sentidos na Assistência Transespecífica em Saúde, Direitos e Cidadania Trans*
}

\begin{abstract}
Resumo: Neste artigo, discutimos o processo de produção de sentidos na assistência transespecífica em saúde e sua relação com os direitos e a cidadania trans*, a partir do diálogo com 11 pessoas, entre usuários(as), médicos(as) e funcionários(as) de um serviço de atenção em saúde para pessoas trans* localizado em uma capital de estado do Sul do Brasil. Os(as) participantes foram entrevistados(as) e essas informações foram analisadas a partir da problematização recursiva. Os resultados organizam-se em torno de duas tramas de sentidos. Por um lado, analisa-se o significado e a potência das demandas das pessoas trans* no campo da assistência transespecífica em saúde, apontando sua relação com uma ética comprometida com o que é excluído pelos regimes de verdade e com a garantia de novos fluxos de inteligibilidade. Por outro lado, analisa-se os requisitos para a constituição do espaço de atenção em saúde para as pessoas trans* como um âmbito para a concretização da cidadania trans*, que se supõe garantir não apenas pelo acesso ao setor da saúde, mas também pelas características da oferta, sua formalização, a manutenção e a institucionalização do cuidado às pessoas trans*.
\end{abstract}

Palavras-chave: Transexualidade, Diversidade de gênero, Acessibilidade aos serviços de saúde, Direitos humanos, Políticas públicas antidiscriminação.

\section{Introducción}

Las recientes investigaciones desarrolladas en Brasil han mostrado las dificultades que enfrentan las personas trans ${ }^{* 1}$ para acceder al sistema de salud y a una asistencia transespecífica que responda y tome en consideración sus necesidades (Cardoso \& Ferro, 2012; Lazcano, 2019; Mujica, 2014; Rocha et al., 2009; Sampaio \& Coelho, 2012; Tagliamento, 2012). Pese a las iniciativas y los innegables avances que se reportan en el ámbito de la salud pública brasileña en los últimos años, fundamentalmente después de su democratización, la demanda de acceso y acogida de las personas trans* en el sector de la salud continúa ocupando un lugar prominente en la agenda de reivindicaciones de los movimientos transfeministas. Esta demanda se articula no solo con la necesidad de democratizar y ampliar el acceso a la asistencia, sino de transformar esos espacios en ámbito de ejercicio transciudadano.

La noción de ciudadanía trans*, proveniente de las teorizaciones en torno a la ciudadanía sexual (Cooper, 2006; Richardson, 2005) y las continuas problematizaciones sobre acceso a derechos en el marco de los estudios de género y LGBTQ (lesbianas, gay, bisexuales, trans, queer), busca ampliar las posibilidades de participación de las personas trans* dey en la esfera pública, tradicionalmente cisnormativa y heterosexualizada. Al reflexionar sobre las condiciones para el ejercicio de la transciudadanía, Jaqueline Gomes de Jesus (2014) apunta que se trata de una ciudadanía que no comienza otorgada por alguien, sino que comienza dentro de las personas trans*, exigida individual y colectivamente. No es, por tanto, una ciudadanía tutelada, sino una ciudadanía conquistada.

Lo anterior implica la creación de mecanismos para que las personas trans* se vuelvan agentes activos en la producción de conocimiento sobre sí y la implementación de formas de participación contrahegemónicas. De ahí la importancia de reflexionar sobre las restricciones conceptuales y políticas de algunos de los instrumentos de garantía de derechos como el Proceso Transexualizador a través del Sistema Único de Salud (SUS), una de las más importantes políticas

\footnotetext{
${ }^{1}$ Teniendo en cuenta que la transexualidad solo puede ser comprendida en su multiplicidad y heterogeneidad, asumimos el término trans* (seguido del asterisco), así como lo hace Platero (2014), para incluir una amplia gama de expresiones de género y formas de autoidentificación, entre ellas, transexual, travesti, transgénero. Enfatizamos con ello el reconocimiento de la diversidad de cuerpos, vivencias e identidades que el término puede acoger. Desde una Epistemología de las Márgenes (Bento, 2017), su empleo es también una forma de rechazo a la idea de identidad común que abarca a los grupos excluidos.
} 
antidiscriminatorias lanzadas en Brasil en las últimas décadas en el ámbito de salud, que condensa y operacionaliza por medio de normativas que la estipulan, las pretensiones de inclusión y acogida de las personas trans*, la humanización de los servicios y la integralidad en la asistencia transespecífica en salud.

No obstante, los autores y las autoras como Arán, Murta y Lionço (2009), Bento (2012), Borba (2014) y Teixeira (2017) discuten la (des)articulación entre sus pretensiones, los referentes en que se basa y su operacionalización práctica, tanto por las estrategias seguidas para su perfeccionamiento/evaluación como en relación a su implementación. Enfatizan sus reflexiones en las implicaciones y los efectos que genera dicha (des)articulación en términos de calidad y eficacia de los modelos de asistencia implementados, así como en función de la mejoría de los indicadores de salud (morbimortalidad, expectativa de vida y otros) de las personas trans*. Además, destacan sus efectos en términos de precarización y vulnerabilidad de las transexistencias.

Flavia Teixeira (2017) pone en diálogo las normativas que aseguran la realización del Proceso Transexualizador por medio del SUS con los procedimientos, protocolos y mecanismos estipulados para su consecución, y discute las paradojas implícitas en la política y su incapacidad para incluir a todas las personas trans* en su diversidad; mostrando que solo a las mujeres trans le son garantizados ciertos accesos y derechos, mientras que a los hombres trans y las travestis le son retirados. La autora problematiza la centralidad de las resoluciones del Consejo Federal de Medicina (CFM) y de los saberes médicos, con gran poder normalizador de los cuerpos. Al mismo tiempo, discute cómo, aun cuando la normativa establece una línea de cuidado para las travestis y transexuales como parte de la salud pública brasileña, esta es fuertemente impactada por la heteronormatividad y el binarismo de género, lo que, junto a la patologización de las transexualidades, acaba por acentuar la precariedad de las transexistencias.

Berenice Bento $(2014,2017)$, en el contexto de los derechos transciudadanos en Brasil, discute la emergencia de un marco legal que tiende a su instauración como una gambiarra ${ }^{2}$ legal. Alude con ello al carácter de fachada de los "arreglos" que en términos legales han sido tradicionalmente prescritos como soluciones, convirtiéndose en mecanismos generadores de una "ciudadanía precaria", que Oliveira (2013) define como "de consolación" al configurar apenas un cuadro de democracia formal.

Con vistas a garantizar el ejercicio de la ciudadanía de las personas trans*, y de que los espacios de cuidado en salud creados para atender a sus demandas constituyan una garantía de ello, partimos del presupuesto de que, si bien es necesario asegurar su acceso mediante instrumentos legales, también es esencial discutir los presupuestos teóricos, epistemológicos y axiológicos, que aseguren la constitución de esos espacios de cuidado en espacios dialógicos y coparticipativos. Tales ideas plantean la necesidad de desestabilización del estatuto epistemológico de las ciencias médicas (Lazcano, 2019).

De ese modo, consideramos importante discutir el proceso de producción de sentidos en torno a la asistencia transespecífica en salud a partir del diálogo con las/os usuarias/os, médicas/os y empleadas/os de un servicio de atención a personas trans* de una capital en la región Sur de Brasil, focalizando en algunas de las categorías y/o presupuestos desde las cuales se piensa su relación con los derechos y la ciudadanía trans*.

\section{Sobre el servicio de atención en salud para personas trans*}

El servicio de atención en salud para personas trans* que trata esta investigación se creó en agosto de 2015 con la finalidad de ofrecer una atención integral en salud a las personas trans* y al género diversas, con demandas asociadas al proceso transexualizador y fundamentalmente a la hormonización. En la región Sur de Brasil es el segundo destinado específicamente a las personas trans*. La atención es realizada por médicos y residentes de la Especialidad de Medicina de Familia y Comunidad de la Secretaría Municipal de Salud (SMS), y desde sus inicios tiene como sede una Unidad Básica de Salud (UBS) del territorio.

Aunque el servicio toma como referente las normativas que autorizan la realización del Proceso Transexualizador por medio del SUS, no consta como uno de los servicios habilitados por las mismas. En función de los niveles de

\footnotetext{
${ }^{2}$ Se usa la palabra gambiarra en portugués de Brasil para describir una "solución improvisada" al resolver un problema o remediar una situación de emergencia. Es una "alternativa" que puede no ser efectiva a largo plazo o resolver apenas una parte del problema, mientras otros aspectos que le son consustanciales no son alcanzados por dicha "solución" o son, incluso, generados por ella.
} 
satisfacción reportados por la comunidad LGBT de la región, la experiencia se inició como un espacio-atendimiento ideado, gestionado y mantenido de manera voluntaria por médicos residentes de esa especialidad. Desde 2017 lo instituyeron como pasantía obligatoria de la residencia médica.

Esta pasantía obligatoria se asoció también al compromiso ético-político de los profesionales involucrados en la experiencia (residentes, preceptores y funcionarios de la SMS vinculados a la Residencia), y su empeño en mantener el servicio funcionando. Esta estrategia respondió, también, a la voluntad de convertir la experiencia en espacio docente-asistencial, como vía de garantizar la capacitación y competencias culturales de los futuros profesionales de la Atención Primaria (APS) del territorio, para acoger y acompañar en este nivel las demandas de las personas trans*. La estrategia, si bien representó una aparente formalización u oficialización de la atención que ya venía siendo realizada, no marcó su instauración como política pública, ni que el servicio fuese contemplado por presupuestos y planes de acción de las instancias de salud en el territorio.

Durante la realización de esta investigación, ningún profesional trans* trabajaba en el equipo. No obstante, la participación de varias activistas trans fue decisiva en la creación del servicio. En los dos primeros años, la comunicación y articulación con liderazgos trans* del territorio fue esencial para su funcionamiento y mejoría. Posteriormente, las activistas trans* participaron en capacitaciones destinadas alos/ as funcionarias de la UBS que acogieron inicialmente el proyecto, a fin de habilitarles para una acogida sensible de las/os usuarias/os. La estrecha comunicación de organizaciones de defensa de los derechos humanos de las personas trans* de la región y el equipo de médicos que realizaba la atención, mostró la potencia del trabajo conjunto y articulado en la construcción de espacios de concreción de derechos.

\section{Pautas metodológicas para la producción de información y presentación de los/as interlocutores/as en la investigación}

La investigación fue aprobada, en agosto de 2017, por el Comité de Ética en Investigación con Seres Humanos y, posteriormente, por la Comisión de
Acompañamiento de Proyectos de Investigación en Salud, perteneciente a la Secretaría de Municipal de Salud (SMS) del lugar donde se desarrolló la investigación. El acceso al campo se inició en septiembre de 2017 y se extendió hasta junio de 2018.

En cuanto a la metodología, este estudio parte de la perspectiva cualitativa. En la investigación se llevaron a cabo entrevistas abiertas con 11 personas ${ }^{3}$.

- Dos médicos preceptores en el momento del acceso al campo: Fabio (hombre cis, 29 años, raza blanca. Fundador del servicio - en ese momento era residente de la Especialidad de Medicina de la Familia y Comunidad) y Leila (mujer cis, 34 años, raza blanca).

- Residente de la Especialidad de Medicina de la Familia y Comunidad: Caio (hombre cis, 24 años, raza blanca).

- Dos funcionarios de la SMS vinculados a la especialidad: Marcos (hombre cis, raza blanca, nivel educativo superior, edad no declarada) y Vinicius (hombre cis, 35 años, raza blanca, nivel educativo superior con formación en Medicina de la Familia y Comunidad).

- Funcionaria de la SMS vinculada a la Gerencia de Atención Primaria, que participó activamente en las discusiones sobre la aprobación e instauración del servicio: Iara (mujer cis, 42 años, raza blanca, formación en enfermería, nivel educativo superior)

- Coordinadora de la Unidad Básica de Salud donde el servicio comenzó a funcionar: Patrícia (mujer cis, 41 años, raza negra, formación en enfermería, nivel educativo superior).

- Usuarios/as/es": Ana (travesti, 23 años, raza blanca, estudiante de maestría y trabajadora sexual), Isabel (mujer trans, 29 años, raza blanca, educación secundaria concluida, trabajadora sexual), Erik (hombre trans, 46 años, raza negra, nivel educativo concluido, estudiante de maestría, coordinador de proyectos en educación en las áreas de derechos humanos, sexualidad y diversidades de género) y Ju (no-binarie, 25 años, raza blanca, nivel educativo, maestría).

Para contactar a los/as participantes se aplicó el recurso de la bola de nieve (Biernacki \&Waldorf, 1981).

\footnotetext{
${ }^{3}$ Para preservar la identidad de los/as participantes se utilizó nombres ficticios en este estudio.

${ }^{4}$ El género declarado se corresponde con las categorías de autoidentificación de cada participante.
} 
El análisis de las informaciones se realizó a partir de la problematización recursiva formulada por Ana María Fernández (2007) que propone "pensar problemas" antes que "aplicar" o "comprobar" sistemas teóricos, procurando hacer de la incomodidad un concepto para que lo invisible opere visibilidad. La problematización recursiva supone, por tanto, construir estrategias de pensamiento que permitan interrogar los problemas que las teorizaciones han hecho posible, interrogar los a priori, pensar en el límite de lo que se sabe, y en base a ello elaborar campos de problemas que permitan nuevas teorizaciones. Como resultado, se identificaron tramas de sentidos que agrupamos por núcleos temáticos, y las que discutimos en este artículo se relacionan a -o (re)significan- una cierta noción de demanda, derecho y ciudadanía, todas en estrecha relación.

\section{Producción de sentidos, política transcrítica y ciudadanía trans*: interlocuciones teóricas para una plataforma de diálogo}

Desde una perspectiva psicosocial crítica, entendemos el proceso de producción de sentidos como como una producción dialógica en la cual participan diversos actores sociales; una coconstrucción que alude a emprendimientos colectivos e interactivos, por medio de los cuales "las personas en la dinámica de las relaciones sociales históricamente datadas y localizadas construyen los términos y, a partir de ellos, comprenden y se posicionan en situaciones cotidianas" (Spink, 2003, p. 16).

Los procesos de producción de sentidos, como parte de los "actos de encuadrar" (normativos y regulatorios), se traducen simultáneamente en "actos de reconocimiento", delineando formas particulares de aprehender, controlar y administrar las vidas de manera diferenciada. Dichos procesos se relacionan, en ese sentido, con la configuración y distribución diferencial de condiciones precarias, entendiéndolas, según Butler (2015), como condiciones políticamente inducidas a través de las cuales "ciertas poblaciones sufren con redes sociales y económicas de apoyo deficientes, y quedan expuestas de forma diferenciada a las violaciones, a la violencia y a la muerte" (p. 46). Por todo ello consideramos esencial puntuar la relación de los procesos de producción de sentidos, las políticas públicas y el ejercicio de la ciudadanía trans*.

Partimos del entendimiento de que existe una dimensión esencial en el proceso de garantía de la transciudadanía, que se relaciona con el lugar de enunciación del derecho, inclusive con su conceptualización como referente para la promoción y concretización de acciones, actuando a favor de la politización de las reivindicaciones de las personas trans*. La ciudadanía sexual y, como parte de ella, la transciudadanía afirma la necesidad de discutir aspectos de una ciudadanía diferenciada y de politizar el género y la sexualidad. Como hemos analizado la ciudadanía se constituye como una noción/estatus generificada, sexualizada, racializada, marcada por la voluntad de reivindicar los derechos tradicionalmente negados a determinados sujetos, con vistas a su incorporación a la sociedad como miembros plenos de derechos, de responsabilidades y destinatarios de reconocimiento social (Evans, 1993; Oliveira, 2013).

Operar con esta noción de ciudadanía permite distinguir y discutir ciertas modalidades de ciudadanía sexual, específicamente de la ciudadanía trans*, que habitan el espacio de las políticas públicas y que acaban reduciendo las formas de participación a aquellas que el Estado, los sectores en el poder y los saberes-poderes disciplinares preestablecen/ permiten al despolitizar los sujetos que las demandan. Además, problematizan las estrategias pautadas por esas instancias para garantizar derechos al pautar espacios y mecanismos de normalización cis ${ }^{5} \mathrm{y}$ heteronormativos, que funcionan como criterios de "asimilación social” y deslegitiman otras expresiones de género no-normativas.

Este análisis problematiza también cómo la vía legislativa -aunque se observen las condiciones de formulación de las normativas- no debe ser tomada como fin último, pues ella en sí misma no agota las necesidades que impone la ciudadanía trans*. Es necesario observar y cuestionar las vías establecidas para la creación de esos marcos legales, las personas involucradas, el origen y la naturaleza de las demandas que ese marco procura solventar, entre otros aspectos.

\footnotetext{
${ }^{5}$ El concepto de cisgeneridad abarca las personas no-transgénero, siendo relativo a la identidad de género de aquellas personas cuya experiencia individual se corresponde con el sexo que se atribuye a ellas en el nacimiento. La cisgeneridad reproduce un ideal de coherencia entre sexo y género que se instituye como criterio de colonización de los cuerpos y pauta para su categorización normativa como normales, saludables o, por el contrario, anormales, enfermas, inferiores (Simakawa, 2015).
} 
Así la transciudadanía discute la emergencia de ciertas nociones elitistas de derechos e interpela a paradoja sobre su garantía en las sociedades modernas, organizadas bajo un orden jurídico que legitima una relación proporcional entre ambos (derechos y orden jurídico). De este modo, por un lado, tenemos la idea de que los derechos se concretan, apenas o en primera instancia, por medio del aparato legal-del cual hacen parte las políticas públicas como instrumentos de los Estados-nación-. Esta idea se asienta en una racionalidad que asocia la emergencia del aparato jurídico a una noción de "avance", de "conquista de derechos", y que supone ser cuestionada como verdad absoluta.

Por otro lado, resulta imposible desconsiderar que, en las sociedades organizadas a partir de y en torno al aparato estatal, el estatus de sujeto es, sin duda, producido en la relación de protección y reconocimiento otorgado por ese dispositivo, por medio de sus políticas y dependiente de ellas. Esta última idea es analizada por Butler $(2006,2015)$ enfatizando la responsabilidad que implica el reconocimiento de la precariedad de la vida humana, de algunas más que otras, produciendo o ampliando su vulnerabilidad como efecto de la segregación social. En este caso, la vulnerabilidad resulta "inducida" por los sistemas políticos cis y heteronormativos, clasistas, racistas, capacitistas y coloniales, que niegan el estatus de sujetos a determinadas poblaciones.

Entendiendo que las herramientas de acción y control de las políticas públicas pueden legitimar también una desigual distribución del duelo, Butler (2015) reflexiona sobre esa compleja y paradójica red de relaciones que se enlaza a una cierta idea de "protección ciudadana". En consecuencia, problematiza el hecho de que sea el Estado Nación la instancia a la cual las personas acuden en búsqueda de protección, cuando es precisamente de él que ciertas poblaciones necesitan ser protegidas.

De este modo, las políticas sociales emergen como un campo de lucha importante, de y contra la vulnerabilidad de las poblaciones. Su desmonte y precario funcionamiento contribuye negativamente a los objetivos de la promoción de la ciudadanía sexual de modo general, instituyendo al mismo tiempo el campo de las políticas públicas como un espacio de disputa por la inteligibilidad social y contra las condiciones de precariedad políticamente inducidas.
Spade (2015), desde una perspectiva política trans* crítica, analiza las limitaciones de la legislación sobre discriminación e indica que para combatirla eficazmente se debe comprender su funcionamiento. El autor subraya que las estrategias de cambio promovidas por las normas jurídicas, en general, no combaten suficientemente el control disciplinar, sino que a veces lo promueven al utilizar los saberes disciplinares normativos y normalizadores para regular prácticas-intervenciones; ante esto cabe problematizar el modo en que pueden asegurar la concreción de derechos.

Como recurso para esa problematización, Spade (2015) parte de un postulado crítico de resistencia que se propone cuestionar por qué la desigualdad continúa y/o aumenta, incluso durante períodos en que se han aprobado políticas para combatir la discriminación y ampliar los accesos. Para ello se basa en una revisión amplia de contextos, prácticas, procesos de creación y establecimiento de las normativas, y tensionamiento de los procesos de normalización que albergan. Así reflexiona sobre cómo actúa el poder ${ }^{6}$ y los sistemas de significación y control, por medio de las políticas creadas para proteger a ciertas poblaciones de sus efectos.

Este análisis del campo de las políticas públicas en salud, del Proceso Transexualizador, por medio del SUS y de la asistencia en salud a las personas trans* permite interrogarnos la forma en que emerge la discriminación mediante los modos de concebir y administrar la salud.

Con base en esta discusión, explicitaremos a continuación dos núcleos de significación que condensan el análisis: el primer núcleo discute la significación y la potencia de las "demandas" de las personas trans* al campo de la asistencia transespecífica, promoviendo su relectura y reinterpretación frente a las necesidades de las mismas en el ámbito de la salud. El segundo se refiere a las exigencias para constituir el espacio de la atención en salud para personas trans* como un ámbito (físico y simbólico) de concretización de derechos ciudadanos.

\section{Las demandas en salud transespecíficas: reflexiones para una (re)politización del término}

Como se ha problematizado en apartados anteriores, la creación de políticas de asistencia sanitaria para personas trans* no es suficiente - aunque

${ }^{6}$ Considerando, a partir de Foucault (2007), que el poder no se encuentra solo en una persona o institución dominante, sino que las tecnologías de poder se distribuyen de diversas maneras. 
necesaria- para garantirles mayor acceso a la salud. Tampoco puede afirmarse que un mayor acceso garantizará, de forma ineludible, la disminución de las desigualdades. Para pensar en estas paradojas, es esencial realizar una lectura atenta de las demandas planteadas por las/os usuarias/os de los servicios, entendiendo que la deslegitimación de su lugar de enunciación como productores de conocimiento, además de constituir una forma de subalternización y/o epistemicídio, atenta contra el ejercicio de la transciudadanía.

Algunos fragmentos de las entrevistas ${ }^{7}$ realizadas resultan especialmente ilustrativos del análisis y la problematización que emprendemos. Una de las usuarias del servicio refiere:

...Si yo llego y veo que no me llaman por mi nombre social... o que es una consulta para estar cuestionando lo que soy, lo que me gusta, lo que quiero... o tratando de IMPONER [énfasis en la palabra] lo que tengo que hacer, sin oír lo que tengo que decir sobre MI CUERPO [énfasis en la expresión], yo me voy, así de simple (Isabel, usuaria).

Otrausuaria, demodosimilar, afirma:“...elprofesional que está allí empeñado en ayudarnos necesita tener esa comprensión de nuestra realidad social, de que es una realidad dada a la violencia, a la discriminación. Si no es sensible a nuestras experiencias, de nada sirve estar alli" (Ana, usuaria). Erik, también usuario, manifiesta al referirse a su experiencia dentro del servicio y con algunos de los profesionales que realizaban la atención: "Ellos me preguntaban de la familia, del trabajo. Era realmente aquella medicina acogedora donde se trata no sólo el usuario, sino todo el entorno. Yo nunca me sentí tan bien acogido dentro del sector de la salud". El mismo usuario afirma en otro momento: "Creo que muchos de nosotros salimos [de las consultas/servicios] con las necesidades no sanadas cuando se prescribe solo la hormonioterapia, porque nuestra demanda es mucho mayor que eso".

Como puede apreciarse, las "demandas" en el contexto de la atención transespecífica en salud emergen como un conjunto de necesidades y expectativas con relación a las mismas, y que suponen ser entendidas necesariamente de un modo abarcador, no restringiéndose a la lectura biomédica que traduce entidades anatomo-fisiopatológicas actuales o potenciales, o a la solicitud de prescripción de procedimientos médico-quirúrgicos, como parecen sugerir algunos sentidos construidos sobre el término en el contexto de la atención transespecífica en salud. La siguiente intervención de una de las usuarias es ilustrativa.

¿Antiguamente qué era la transexualidad? Ese apuro, esa euforia por mudar el cuerpo... pero hoye en día no, hoy en día muchas personas que se colocan como trans ya no luchan por un cuerpo idealizado, muchas tampoco se hormonizan... Para que veas cómo las demandas se diferencian, algunos grupos quieren tratamiento hormonal, otros no, algunos grupos quieren cambios por medio [de] cirugías, otros quieren ambas cosas, o ninguna (Ana, usuaria).

Aunque obviamente la familiarización y el dominio de los profesionales con los protocolos médicos para la hormonización (y otros métodos) sean importantes y se relacionen con los niveles de satisfacción reportados por los usuarios/as con el servicio, se trata de una población cuyas expectativas se sitúan, fundamentalmente, en la dimensión de los vínculos. Dichas expectativas confrontan la ética del cuidado y los modelos tradicionalmente implementados, mostrando que la garantía del derecho ciudadano a la salud está relacionada no solo a la posibilidad de acceder a los espacios de cuidado, sino también a su capacidad para acoger y permitir que la persona usuaria participe en del proceso de construcción de su salud.

Esa noción de demanda que se articula mediada por una pretensión de no subalternización (Spivak, 2010) convoca al sujeto a hablar y a las ciencias biomédicas a entender al sujeto y acogerle. Incita, también, a repensar los fundamentos ético-políticos del cuidado y de la producción de la salud humana con base en mecanismos coparticipativos, que no sustraigan u omitan al sujeto. Ello resulta consubstancial a la idea de transciudadanía, apuntada por Jesus (2014), quien afirma como uno de sus elementos distintivos el hecho de ser conquistada, nunca tutelada, parte de un proceso que se inicia dentro de las personas trans* y que se exige de manera individual y colectiva. La autora coloca de este modo la coparticipación en la producción de esa noción de derecho como una de sus dimensiones esenciales, que emerge necesariamente coconstruida. Lo expuesto convoca a la creación de espacios dialógicos y a pensar el acto de

\footnotetext{
${ }^{7}$ Los fragmentos de las entrevistas fueron traducidos por las autoras.
} 
cuidar con base en formas de participación contrahegemónicas, que reconozcan el derecho a decidir sobre sí de las personas trans*.

Frente a esas evidencias, algunos profesionales participantes de la investigación constatan igualmente la existencia de un campo de problemas en torno a la asistencia y la satisfacción de los usuarios con ella. Como parte de sus valoraciones argumentan: “... acompañarles [a las personas trans*] en el proceso de hormonización es esencial... y atender todas sus demandas... pero la posibilidad de ofrecer un tratamiento humano y acogedor ¡es esencial!' (Caio, médico residente de pasantía en servicio).

Otros, de modo similar, afirman:

Es necesario un vínculo amigable, donde la recepcionista, los funcionarios, todos tengan esa discusión internamente, entiendan la necesidad de colocarse más allá de sus propias concepciones y brindar un atendimiento independiente de lo que cada persona piensa . . . En este, como en todos los casos, el profesional de la salud no puede imponer su visión cultural, sus valores morales y culturales (Marcos, funcionario de la SMS).

Es esencial la implementación de una lógica no medicalizadora... que enseña que en verdad lo que necesitan [a las personas trans*] es ser acogidas, es ver su demanda acogida ... Esa es una percepción que creo que tiene relación con esa lógica de cuidado integral... Cuando los residentes pasan por aquí, nosotros trabajamos mucho esa cuestión de la no patologización, y de entender que el sufrimiento psíquico que muchas veces está presente en esas personas está mucho más relacionado a la transfobia, que a cualquier situación de enfermedad que aquella persona padece (Fabio, médico preceptor del servicio y fundador de este).

Se confirma aquí una producción de sentido en torno a la asistencia transespecífica en salud que la asocia con un acto de cuidado que no debería (como principio para una buena práctica) situar el objetivo terapéutico en la medicalización del sujeto, sino en la forma en que, a través de ella, se garantiza el derecho a existir como personas trans*, considerando los cuerpos no solo como seres biológicos, sino como sujetos de derechos. Tales garantías emergen inscritas en una secuencia de actos (procedimientos, formas de interacción, etc.) no patologizantes y contrahegemónicos, definitorios de la posibilidad de "acoger" a través de los vínculos establecidos.

Entendemos así que, en el contexto de la asistencia en salud, la demanda es siempre más amplia y compleja que el motivo de consulta. Esto supone pensar en la demanda como una categoría política que pone en cuestión la integralidad del cuidado y la tutela biomédica, al revelar el conjunto de las necesidades con que la persona llega al servicio de salud y sus expectativas en la dimensión relacional: asociadas al respeto al nombre social, a la participación en los espacios de consulta y la construcción de su salud, así como en relación con la habilitación de la consulta como un espacio de diálogo no normativo.

Esta noción de demanda, en referencia a lo expresado por las/os participantes de esta investigación, emerge también como una forma de resistencia a la precarización de las trans* existencias y de las políticas dirigidas a la garantía de sus derechos, entendiendo, a partir de Butler (2017), que la resistencia se configura con un acto de rechazo a la precarización. Se trata así de un modo de subversión de la norma que repolitiza la situación de vulnerabilidad de los cuerpos humanos, al mismo tiempo que aprovecha su interdependencia como condición de posibilidad para la "responsividad", tomando el cuerpo como locus de la acción política.

Las "demandas" que las personas trans* llevan a los servicios de salud interpelan el poder normativo que le somete al sujeto, a la vez que inauguran la vuelta reflexiva del propio sujeto sobre sus derechos, induciendo a la agencia. Así como lo sostiene la usuaria trans (Isabel) cuando decide, frente a la precarización de los vínculos: “... me voy, así de simple...”, su retirada no se configura como un acto de renuncia, sino de resistencia a las características y los efectos de subalternización que esos vínculos albergan y/o promueven. De esta forma, la demanda viene a expresar también lo que Butler (2001) entiende en términos de un dilema tropológico: el poder que inaugura/funda el sujeto es condición de subordinación y al mismo tiempo precondición de la acción, de la resistencia al poder que lo sujeta.

Los sentidos producidos configuran, por tanto, una noción de demanda que, en el contexto de la asistencia transespecífica, se expresa en relación a una ética comprometida con: a) lo es excluido por los regímenes de verdad establecidos por las ciencias biomédicas; b) las formas de garantía el derecho transciudadano 
a una atención en salud que respete y tome en consideración las particularidades de las personas trans*, institucionalizando la libre expresión de género y; c) la instauración de nuevos flujos de inteligibilidad.

Aunque estas problemáticas pueden ser extensivas a la atención y cuidado dispensado a toda la población, en el caso de las personas trans* emergen como una condición esencial para garantizar la construcción de más equidad en los servicios y universalidad en el acceso. Constituyen además una pauta para el perfeccionamiento del acto de cuidar y presupuesto para su constitución como espacio de concreción de derechos.

En relación con esa idea, se configura además la integralidad de la atención como consubstancial al constatarse una producción de sentidos que ve en ella un espacio de amplia cobertura a las necesidades en salud de las personas trans*, no equiparable, por tanto, al proceso transexualizador. Mientras este último se asocia al conjunto de procedimientos que se inscriben en la materialidad del cuerpo y procuran su modificación de acuerdo con la expresión de género con que cada persona se identifica, la asistencia transespecífica en salud se concibe, además, enfocada en la garantía de derechos en todas sus fases. En ese sentido, constituye un espacio de atención dialógico y más horizontal por las formas de relación médico-usuario, lo que posibilita una amplia cobertura de atención, con acceso a diversos procedimientos, especialistas y medicamentos según las necesidades de cada sujeto. A partir de los sentidos producidos, la asistencia transespecífica en salud se configura como un espacio sensible a las particulares demandas de las personas trans*, que responde a sus especificidades sin segregarlas.

Esa problematización sobre la integralidad del cuidado revela una dimensión de la integralidad tradicionalmente no considerada como parte de ella: la necesidad de una cohabitación no hegemónica de los espacios y modos de cuidar. Lo que apunta a la coparticipación y la simetría como sus atributos (no solo importa quién participa, sino cómo participa). Ambos atributos y desafíos, a su vez, implican la desestabilización del estatuto epistemológico de las ciencias médicas, tradicionalmente prescriptivo y basado en una ética universalizante.

Finalmente, podemos concluir que tanto la necesidad de una lectura interseccional y localizada del sujeto trans* como de sus demandas se erigen como requisitos para atención trans-situada y como mecanismo de garantía de derechos.

\section{La asistencia transespecífica en salud y los servicios para personas trans* como concretización de derechos ciudadanos}

Ya en el caso del servicio analizado en esta investigación, el hecho de configurarse como una asistencia garantizada a través de un espacio-atención, creado y sostenido por personas comprometidas/sensibles con la garantía del acceso a la salud y los derechos de las personas trans*, resulta una estrategia que merece amplio reconocimiento. Al mismo tiempo se vuelve incapaz de constituir una garantía de ciudadanía para las personas trans*, sobre todo en contextos sociopolíticos inestables, donde los propios vínculos formales son desechos; los presupuestos, arbitrariamente recortados; y los derechos, retirados. Al respecto lo plantea una de las participantes:

Mi intención aquí es criticar el sistema y no a quienes se organizaron para hacer el proyecto del "ambulatorio" funcionar... Yo sinceramente no veo el "ambulatorio" como un avance en los servicios de salud. Primero porque no es iniciativa del Estado, sino un favor que médicos residentes están haciendo... Punto dos: no tenemos acceso a las hormonas por el SUS, derecho este que solo se concreta a partir de mucha lucha con acciones judiciales... En ese escenario, el "ambulatorio" solo sirve a las personas trans y travestis que tienen condiciones de pagar por las hormonas, haciendo con que personas T pobres, además de marginalizadas y con vidas precarias, no tengan acceso a los medicamentos que necesitan... Entonces es así, no es una política, porque no está beneficiándonos, y digo beneficio en cuanto reparación, no así de privilegio (Ana, usuaria, travesti).

En relación con la discusión anterior, comprender la asistencia transespecífica como un derecho concretizado de manera parcial es otra de las tramas de significación en torno a este tema que emergió del encuentro con las/os participantes. Esto se asocia a las condiciones de su creación y manutención.

$\mathrm{Al}$ reflexionar sobre las condiciones de instauración del servicio, Fabio, uno de los profesionales vinculado al mismo y uno de sus fundadores refiere que "la idea consiguió avanzar" y el servicio se creó, porque "no generó ningún costo extra para la red", y entiende que "hoy no tiene resistencia porque continúa siendo un espacio que no genera costos". 
La existencia del servicio de asistencia transespecífica se basa, por lo tanto, en una relación con personas políticamente comprometidas con la causa trans o militantes, pero no refleja la existencia de una línea de trabajo instituida por la instancia institucional. Así evidencia un contexto de reconocimiento parcial de derechos y necesidades en salud de las personas trans*, lo que limita su comprensión como concretización de una política pública.

No obstante, los impactos del fuerte personalismo que caracterizan la experiencia de instauración del servicio pueden ser analizados desde varias perspectivas. Por un lado, se asocia a la persistencia de ciertas condiciones de precariedad, generadas por un tipo de vínculo que se configura como inestable, dependiente fundamentalmente de personas que se ponen a disposición para hacerlo acontecer y no de políticas o estrategias creadas, implementadas y respaldadas por planes, estrategias y presupuestos, para atender a las necesidades en salud de las personas trans*. Por otro lado, emerge como condición favorable para que personas sensibles y comprometidas con la causa se vinculen al servicio, garantizando la realización de atención trans-situada.

Cabe interrogarse en ese contexto analítico sobre la significación de la necesidad de "reparación" mencionada por la usuaria y la instauración del servicio como un "proyecto solidario". En el diálogo con las demandas de los/as usuarios/as y la perspectiva trans crítica, esas implicaciones pueden ser pensadas en la dimensión de la responsabilidad institucional y gubernamental de la atención, que traspasa algunas responsabilidades individuales (en forma de "buena voluntad") y que no pueden ser suplidas por ellas.

Al reflexionar sobre las condiciones de surgimiento del servicio y su instauración (dos años después) como pasantía obligatoria de la Residencia, Leila (médica preceptora) afirma: "Hoy podemos decir que la atención [a las/os usuarias/os] está más estable, pero la realidad de Brasil es muy compleja para dar cualquier seguridad con relación al futuro... aún conseguimos asumirla, pero no sé hasta cuándo...." De modo similar Erik (usuario) refiere: "Al mismo tiempo que me siento satisfecho y feliz por su existencia, me causa angustia el hecho de que sea algo provisional, de que el equipo cambie constantemente, de que no tenga apoyo de la Municipalidad, de que no haya un mejor acompañamiento de ese proyecto".
En ese contexto, la relación ético-política del personal que realiza los servicios y su afinidad con la causa trans*, aunque es esencial a los objetivos de acogimiento y humanización de los servicios, resulta condición necesaria pero no suficiente. Las intervenciones citadas consideran el servicio como una "gambiarra" (Bento, 2014), que no ha sido instaurado como una política pública real y concretizado a partir de acuerdos que a largo plazo se muestran insuficientes para garantizar y consolidar los derechos ciudadanos de las personas trans*, contribuyendo a la precarización de la asistencia sanitaria dirigida a estos grupos. Es esencial considerar, en relación a las demandas de las personas involucradas y las características del establecimiento de servicios como ya se apuntó, que la transciudadanía supone garantizarse no solo por el acceso al sector de la salud, sino también por las características de la formalización y manutención de la oferta. Para la "reparación" de las personas trans* y la garantía de derechos, es necesario trabajar la transformación de las lógicas actuales del Estado para garantizarles la igualdad y los derechos sociales.

Se constata la emergencia de pautas de funcionamiento que actúan deslegitimando la transgeneridad como foco de la atención y acciones en salud. La instauración de prioridades emerge como un proceso marcado, de esta forma, por normas de reconocimiento que subalternizan las demandas de atención transespecífica en salud y al propio sujeto trans*. La condición precaria se verifica aquí como un punto políticamente inducido (Butler, 2015), a través del cual las personas trans* son vulnerabilizadas por redes sociales y económicas de apoyo deficientes. La garantía de una asistencia transespecífica en ese contexto y en el diálogo con la ciudadanía trans* resulta congruente con lo que Butler (2015) analiza como un "ejercicio performativo del derecho a aparecer" (p. 31), una reivindicación corporificada de una vida más vivible, que observa en la ocupación política de los espacios un recurso contra la precarización.

\section{Consideraciones finales}

En este artículo discutimos el proceso de producción de sentidos sobre la asistencia transespecífica en salud y su relación con los derechos y la ciudadanía trans*.

La (re)producción de la transciudadanía en el ámbito de la salud por medio de una asistencia transespecífica es el resultado no solo de la creación de espacios para un acceso a la asistencia que reproduce 
los poderes hegemónicos, sino también de la desestabilización de las normas. La demanda de las personas trans* reclama su conversión en un ámbito que reconozca y potencie la autonomía de las personas trans*, y no su represión a partir de criterios normativos disciplinares o de normativas que institucionalizan un cuidado que no presta atención en el sujeto y enfatiza el cumplimiento de protocolos y procedimientos.
Al repolitizar el acto de cuidar, es esencial problematizar lo que se potencializa en esos espacios, cuáles las narrativas que se legitiman, cuáles demandas se reivindican y cómo se hace. Es esencial comprender las nociones y modelos de cuidado con que se opera en la dinámica de la asistencia en salud dispensada a las personas trans*, de modo que promuevan un cuidado que no quede atrapado en las normativas que lo institucionalizan.

\section{Referencias}

Arán, M., Murta, D., \& Lionço, T. (2009). Transexualidade e saúde pública no Brasil. Ciência \& Saúde Coletiva, 14(4), 1141-1149. http://doi.org/10.1590/S1413-81232009000400020

Bento, B. (7 jun. 2012). Carta-desabafo. Estado Laico RJ.http://estadolaicorj.blogspot.com.br/2012/06/berenicebento-carta-desabafo-sobre-o.html

Bento, B. (2014). Nome social para pessoas trans: Cidadania precária e gambiarra legal. Contemporânea, 4(1), 165-182. http://www.contemporanea.ufscar.br/index.php/contemporanea/article/view/197/101

Bento, B. (2017). Transviad@s: Gênero, sexualidade e direitos humanos. Editora da UFBA.

Biernacki, P., \& Waldorf, D. (1981). Snowball sampling: Problems and techniques of chain referral sampling. Sociological Methods \& Research, 10(2), 141-163. https:// doi.org/10.1177/004912418101000205

Borba, R. (2014). (Des)aprendendo a "ser": trajetórias de socialização e performances narrativas no Processo Transexualizador [Tesis de doctorado, Universidad Federal do Rio de Janeiro].

Butler, J. (2001). Mecanismos psíquicos del poder: Teorías sobre la sujeción. Ediciones Cátedra.

Butler, J. (2006). Vida precaria: El poder del duelo y la violencia (1ra. ed.). Paidós.

Butler, J. (2015). Quadros de guerra: Quando a vida é passivel de luto?. Civilização Brasileira.

Butler, J. (2017). Cuerpos aliados y lucha política: Hacia una teoría performativa de la asamblea. Paidós.

Cardoso, M., \& Ferro, L. F. (2012). Saúde e população LGBT: Demandas e especificidades em questão. Psicologia: Ciência e Profissão, 32(3), 552-563. http://doi.org/10.1590/S1414-98932012000300003

Cooper, D. (2006). Ative citizenship and the governmentality of local lesbian and gay politics. Political Geography, 25(8), 921-943. http://doi.org/10.1016/j.polgeo.2006.08.009

Evans, D. T. (1993). Sexual citizenship: The material construction of sexualities. Routledge.

Fernández, A. M. (2007). Las lógicas colectivas: Imaginarios, cuerpos y multiplicidades. Biblos.

Foucault, M. (2007). Microfísica do poder (24a ed.). Edições Graal.

Jesus, J. G. de. (2014). Transfeminismo: Teorias e práticas. Metanoia.

Lazcano, C. (2019). Humanizando, acolhendo, (trans)formando: Produção de sentidos em torno da assistência transespecífica em saúde [Tesis de doctorado, Universidad Federal de Santa Catarina]. Repositório Institucional UFSC.

Mujica, A. M. (2014). Experiências de atenção à saúde e percepções das pessoas transgênero, transexuais e travestis sobre os serviços públicos de saúde em Florianópolis/SC, 2013-2014 [Disertación de maestría, Universidad Federal de Santa Catarina]. Repositório Institucional UFSC.

Oliveira, J.M. (2013). Cidadania sexual sob suspeita:Uma meditação sobre as fundações homonormativas e neo-liberais de uma cidadania de "consolação". Psicología \& Sociedade, 25(1), 68-78. http://doi.org/10.1590/S0102-71822013000100009

Platero, R. (Lucas). (2014). Trans*exualidades: Acompañamiento, factores de salud y recursos educativos. Bellaterra.

Richardson, D. (2005). Desiring sameness? The rise of a neoliberal politics of normalisation. Antipode, 37(3), 515-535. https://doi.org/10.1111/j.0066-4812.2005.00509.x

Rocha, K. B., Barbosa, L. H. R., Barboza, C. Z., Calvetti, P. Ü., Carvalho, F. T., Cerqueira-Santos, E., Hermel, J. S., \& Moura, A. S. (2009). La atención a la salud en Brasil a partir de la percepción de travestis, transexuales y transgéneros. Forum: Qualitative Social Research, 10(2). https:// doi.org/10.17169/fqs-10.2.1302 
Sampaio, L. L.P., \&Coelho, M.T.A.D. (2012).Transexualidade:Aspectos psicológicose novas demandas ao setor saúde. Interface - Comunicação, Saúde, Educação, 16(42), 637-649. http://doi.org/10.1590/S1414-32832012000300005

Spade, D. (2015). Una vida "normal": Violencia administrativa, políticas trans crítica y los límites del derecho. Ediciones Bellaterra.

Spivak, G. (2010). Pode o subalterno falar? Editora da UFMG.

Spink, M. J. (2003). Psicologia social e saúde. Editora Vozes.

Tagliamento, G. (2012). (In)Visibilidades caleidoscópicas: A perspectiva das mulheres trans sobre o seu acesso à saúde integral [Tesis de doctorado, Instituto de Psicología de la Universidad de São Paulo]. Biblioteca Digital de Teses e Dissertações da USP.

Teixeira, F. (2017). (Des)engano: Revisando as portarias do processo transexualizador no SUS. In A. P. Uziel \& F. Guilhon (Org.), Transdiversidades: Práticas e diálogos em trânsitos (pp. 315-354). Editora da UERJ.

Simakawa, V. V. (2015). Por inflexões decoloniais de corpos e identidades de gênero inconformes: Uma análise autoetnográfica da cisgeneridade como normatividade [Disertación de Maestría, Universidad Federal de Bahia]. Repositório Institucional UFBA.

\section{Claudia Leticia Lazcano}

Doutora em Psicologia pela Universidade Federal de Santa Catarina (UFSC), Florianópolis - SC, Brasil. Professora do curso de Psicologia da Faculdade Cesusc, Florianópolis - SC, Brasil.

E-mail: claudiapsicocuba@gmail.com

(1) https://orcid.org/0000-0001-6805-7984

Maria Juracy Filgueiras Toneli

Doutora em Psicologia. Professora titular do Programa de Pós-Graduação em Psicologia da Universidade Federal de Santa Catarina (UFSC), Florianópolis - SC, Brasil. Bolsista PQ 1B/CNPq.

E-mail: juracy.toneli@gmail.com

(1) https://orcid.org/0000-0002-9311-5020

Endereço para envio de correspondência:

Complexo de Ensino Superior de Santa Catarina (Cesusc). Rodovia SC 401, St. Antonio de Lisboa. CEP: 88.050-

001. Florianópolis - SC. Brasil.

Recebido 05/11/2019

Aceito 23/12/2020

Received $11 / 05 / 2019$

Approved 12/23/2020

Recibido 05/11/2019

Aceptado 23/12/2020

Cómo citar: Lazcano, C. L., \& Toneli, M. J. F. (2022). Producción de sentidos sobre asistencia transespecífica en salud, derechos y ciudadanía trans*. Psicologia: Ciência e Profissão, 42, 1-12. https://doi.org/10.1590/1982-3703003230748

How to cite: Lazcano, C. L., \& Toneli, M. J. F. (2022). Production of meanings in trans-specific assistance for health, rights, and trans* citizenship. Psicologia: Ciência e Profissão, 42, 1-12. https://doi.org/10.1590/1982-3703003230748

Como citar: Lazcano, C. L., \& Toneli, M. J. F. (2022). Produção de sentidos na assistência transespecífica em saúde, direitos e cidadania trans*. Psicologia: Ciência e Profissão, 42, 1-12. https://doi.org/10.1590/1982-3703003230748 